\title{
Liposome-based immunotherapy against autoimmune diseases: therapeutic effect on multiple sclerosis
}

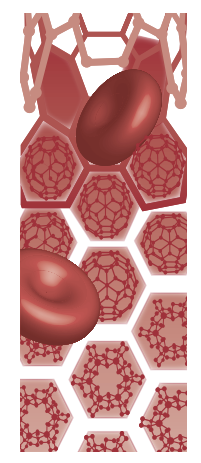

\begin{abstract}
Aim: Based on the ability of apoptosis to induce immunological tolerance, liposomes were generated mimicking apoptotic cells, and they arrest autoimmunity in Type 1 diabetes. Our aim was to validate the immunotherapy in other autoimmune disease: multiple sclerosis. Materials \& methods: Phosphatidylserine-rich liposomes were loaded with disease-specific autoantigen. Therapeutic capability of liposomes was assessed in vitro and in vivo. Results: Liposomes induced a tolerogenic phenotype in dendritic cells, and arrested autoimmunity, thus decreasing the incidence, delaying the onset and reducing the severity of experimental disease, correlating with an increase in a probably regulatory CD25+ FoxP3- CD4 $4^{+} \mathrm{T}$-cell subset. Conclusion: This is the first work that confirms phosphatidylserine-liposomes as a powerful tool to arrest multiple sclerosis, demonstrating its relevance for clinical application.
\end{abstract}

First draft submitted: 2 December 2016; Accepted for publication: 17 March 2017; Published online: 18 May 2017

Keywords: autoimmunity • experimental autoimmune encephalomyelitis • immunotherapy - liposomes $\bullet$ multiple sclerosis

Liposomes are small vesicles with an aqueous core encapsulated by a lipid bilayer that constitute a promising drug delivery system [1]. Liposome formulations have progressed and they are still improving [2,3], and currently they represent an innovative area of great scientific interest due to their wide variety of potential applications in biomedicine. Liposomes protect encapsulated molecules against degradation and can be designed to deliver them into specific target cells [4]. Various types of liposomes are used clinically as vehicles for drugs and vaccines [5]. Liposomes can be designed for multiple purposes, including immune modulation to enhance or inhibit a specific response [6].

We recently developed a liposome-based immunotherapy - inspired by the features of apoptotic cells $[7]$ - to re-establish tolerance to self and avoid autoimmune diseases [8]. These liposomes are rich in phosphatidylserine (PS), a phospholipid membrane com- ponent that is exposed in apoptotic cells and modulates immune responses [9], and are loaded with self-peptides - named autoantigens - as the selective molecule for tolerance induction. PS is recognized by membrane receptors of antigen presenting cells - mainly dendritic cells (DCs) - acting as an 'eat me' and 'tolerate me' signal, which allows the encapsulated autoantigen to be presented in a tolerogenic manner. Working synergistically, PS-liposomes and encapsulated autoantigens display a big translational potential in pathologies that require the re-establishment of immune tolerance.

Autoimmune diseases are caused by the selective destruction of the host's own cells by autoreactive $T$ lymphocytes that recognize autoantigens. There are nearly 100 autoimmune diseases, affecting around 3-5\% of the population [10]. There is no cure for any of them and their etiology is unknown. Moreover, incidence is increasing in the last
Irma Pujol-Autonell', Maria-Jose Mansilla', Silvia Rodriguez-Fernandez', Mary Cano-Sarabia², Juan Navarro-Barriuso', RosaMaria Ampudia', Aleix Rius', Sonia Garcia-Jimeno², David Perna-Barrull', Eva MartinezCaceres', Daniel Maspoch ${ }^{2,3}$ \& Marta Vives- $\mathrm{Pi}^{\star 1,1,4}$

'Immunology Division, Germans Trias i Pujol University Hospital \& Research Institute, Department of Cellular Biology, Physiology \& Immunology, Autonomous University of Barcelona, 08916 Badalona, Spain

${ }^{2}$ Catalan Institute of Nanoscience \& Nanotechnology (ICN2), CSIC \& The Barcelona Institute of Science \& Technology, 08193 Bellaterra, Barcelona, Spain

${ }^{3}$ Institucio Catalana de Recerca i Estudis Avançats (ICREA), 08010 Barcelona, Spain

${ }^{4}$ CIBER of Diabetes \& Associated Metabolic Diseases (CIBERDEM), ISCIII, Madrid, Spain

*Author for correspondence: Tel.: +34934978666 mvives@igtp.cat 
years [11]. The current therapies for autoimmune diseases are not enough effective and cause side effects, such as immunosuppression. Therefore, antigen-specific, simple and safe approaches to recover self-tolerance are required to arrest autoimmunity and to allow target tissue regeneration. In our previous study, an innovative liposome-based immunotherapy - mimicking apoptotic $\beta$ cells to induce self-tolerance - arrested autoimmunity in Type 1 diabetes (T1D) [8]. Considering the potential of liposomes as immunomodulators, we aimed to develop PS-liposomes for another autoimmune disease, multiple sclerosis (MS), to validate their tolerogenic power.

MS is a chronic autoimmune disease of the CNS in which several myelin peptides become the target autoantigens of autoreactive T cells [12]. Genetic and environmental factors contribute to the etiology of the disease but the triggering factor is not known. The autoimmune attack involves autoreactive $\mathrm{T}$ and B cells [13]. Eighty percent of individuals with MS initially develop a clinical pattern with periodic relapses followed by partial or full remission of the symptoms, called relapsing-remitting MS [14]. This disease is characterized by widespread inflammation, multifocal demyelination and axonal loss. The current treatments are systemic immunointerventions, with important side effects [15]. In this context, PS-liposomes loaded with an autoantigen of the disease, the myelin-oligodendrocyte glycoprotein peptide 40-55 (MOG $\left.{ }_{40-55}\right)$, have been proved as a potential antigen-specific therapy for MS patients.

For this purpose, we evaluated the in vivo efficacy of antigen-loaded PS-liposomes in the animal model of MS, the experimental autoimmune encephalomyelitis (EAE). EAE is an immune-mediated disease that reproduces the main clinical and histopathological characteristics of the human disease. It is induced by the administration of myelin antigens. The most frequent EAE model used is C57BL/6 mice injected with the $\mathrm{MOG}_{40-55}$ peptide [16,17], and it has been the major tool to understand the mechanism involved in MS pathogenesis, as well as to test therapies for the human disease. To determine the effect of a therapy in EAE, three different approaches can be used: preventive (before immunization), preclinical (postimmunization) and therapeutic (after the onset of the disease) [17]. Using EAE preclinical approach, we report here the beneficial effect of liposomes rich in PS and loaded with MOG peptide. This work demonstrates that a simple apoptosis-based strategy has the inherent potential of stopping an ongoing autoimmune reaction such as the EAE, validating the immunotherapy and confirming its potential to arrest autoimmunity.

\section{Materials \& methods}

Mice

Wild-type C57BL/6 inbred mice were purchased from Envigo Rms Spain SL (Sant Feliu de Codines, Barcelona, Spain) and housed at the Animal House facility at the Germans Trias i Pujol Research Institute. Mice were maintained under conventional conditions in a temperature and humidity-controlled room with 12-h light/12h dark cycle, with standard chow diet (Teklad Global 14\% Protein Rodent Diet, Envigo) and water provided ad libitum. Only females were used in this study.

\section{Liposomes \& peptides}

MOG $_{40-55}$ peptide (YRSPFSRVVHLYRNGK, Immunostep, Salamanca, Spain), >95\% pure, was chosen because it is a target epitope in MS patients [18]. Liposomes were composed of 1,2-dioleoyl-sn-glycero3-phospho-L-serine (sodium salt) (DOPS, Lipoid, Steinhausen, Switzerland) and 1,2-didodecanoyl-snglycero-3-phosphocholine (DLPC, Lipoid), and cholesterol ( $\mathrm{CH}$, Sigma Aldrich, MO, USA). Liposomes were prepared using the thin film hydration method from a lipid mixture of DOPS, DLPC and $\mathrm{CH}$ at 1:1:1.33 molar ratio, respectively, as described [19], under sterile conditions and at a final concentration of $30 \mathrm{mM}$. Lipids were dissolved in chloroform and the solvent was removed by evaporation under vacuum and nitrogen. The lipids were hydrated with the appropriate buffer (phosphate-buffered saline [PBS], $0.5 \mathrm{mg} / \mathrm{ml}$ solution of $\mathrm{MOG}_{40-55}$ peptide) and the liposomes obtained were homogenized to $1 \mu \mathrm{m}$ by means of an extruder (Lipex Biomembranes, Vancouver, Canada). Encapsulation efficiencies (EE) were calculated according to the equation $\mathrm{EE}(\%)=\left[\left(\mathrm{C}_{\text {peptide, total }}-\mathrm{C}_{\text {peptide, out }}\right) / \mathrm{C}_{\text {peptide, total }}\right] \times 100$, where $\mathrm{C}_{\text {peptide, total }}$ is the initial MOG is $_{40-55}$ peptide concentration and $\mathrm{C}_{\text {peptide, out }}$ is the concentration of nonencapsulated peptide. To measure the $\mathrm{C}_{\text {peptide, out }}$, liposome suspensions were centrifuged at $110,000 \times g$ for $30 \mathrm{~min}$ at $10^{\circ} \mathrm{C}$. The concentration of nonencapsulated peptide was assessed in supernatants by PIERCE BCA protein assay kit (Thermo Fisher Scientific Inc., IL, USA). In addition to PS-rich liposomes loaded with MOG (PSMOG-liposomes), empty liposomes were generated as controls (PS-liposomes). Particle size distribution and stability - expressed as $\zeta$ potential - were measured by dynamic light scattering using Malvern Zetasizer (Malvern Instruments, Malvern, UK) in undiluted samples. Liposome morphology and lamellarity were examined by cryogenic transmission electron microscopy in a JEOL-JEM 1400 microscope (Jeol Ltd., Tokyo, Japan).

\section{DC generation}

DCs were differentiated from bone marrow progenitor cells in supplemented RPMI-1640 culture medium 
(Biowest, Nuaillé, France) containing 10\% heat-inactivated fetal bovine serum (Gibco, Invitrogen, CA, USA), $100 \mathrm{U} / \mathrm{ml}$ penicillin (Normon SA, Madrid, Spain), $100 \mathrm{mg} / \mathrm{ml}$ streptomycin (Reig Jofre, Sant Joan Despi, Spain), $2 \mathrm{mM}$ glutamine (Sigma), $1 \mathrm{mM}$ sodium pyruvate (Gibco), $50 \mu \mathrm{M}$ beta-mercaptoethanol (Sigma) and mouse GM-CSF (1000 U/ml; Prospec, Rehovot, Israel), as previously reported [20]. DC purity was assessed by CD11c-PE-Cy7 staining (BD Biosciences, CA, USA) and cell viability was determined by annexin $\mathrm{V}-\mathrm{PE}$ and 7-AAD staining (BD Biosciences) by flow cytometry (FACS Canto II, BD Biosciences).

\section{Liposome capture by DCs}

To determine whether liposome capture by DCs takes place by phagocytosis, DCs were co-cultured with 100 $\mu \mathrm{M}$ fluorescence labeled PS-liposomes (PS-lipo-OG488; Oregon green 488 DHPE, Invitrogen, CA, USA) during $5 \mathrm{~min}$ to $6 \mathrm{~h}$ at 37 and $4^{\circ} \mathrm{C}$. Cells were extensively washed in PBS to remove all liposomes attached to the cell membrane. Liposome endocytosis was determined by flow cytometry (FACSCanto II, BD Biosciences).

\section{Assessment of DCs phenotype after liposome uptake}

DCs were co-cultured with $1 \mathrm{mM}$ empty liposomes (PS-DCs), liposomes loaded with MOG peptide (PSMOG-DCs) or the equivalent amount of MOG peptide (MOG-DCs) during $24 \mathrm{~h}$, and their phenotype was analyzed by flow cytometry (FACSCanto II, BD Biosciences). As controls, DCs were either cultured in basal conditions to obtain immature DCs (iDCs) or with lipopolysaccharide (100 ng/ml; Sigma) for $24 \mathrm{~h}$ to obtain mature DCs. DCs were stained with monoclonal antibodies to CD11c-PE-Cy7, CD40-APC, CD86-PE and class II major histocompatibility complex (MHC) I-A/I-E-FITC (BD Biosciences). Corresponding fluorescence minus one staining was used as control. Data were analyzed using FlowJo software (Tree Star, OR, USA).

\section{Prostaglandin E2 quantification}

The production of prostaglandin $\mathrm{E}_{2}\left(\mathrm{PGE}_{2}\right)$, a potent immunoregulator responsible for the immunosuppressive mechanism of apoptotic cells, was assessed by ELISA (PGE 2 EIA Kit-Monoclonal; Cayman Chemicals, MI, USA), in supernatants obtained from DC cultures, $24 \mathrm{~h}$ after liposome capture. LOD: 80\% $\mathrm{B} / \mathrm{B}_{0}$ : $15 \mathrm{pg} / \mathrm{ml}$. Sensitivity: 50\% B/B : $50 \mathrm{pg} / \mathrm{ml}$. Results were expressed as pg of $\mathrm{PGE}_{2} / 10^{6}$ cells.

\section{Disease induction \& clinical follow-up in the EAE model}

For the induction of EAE, C57BL/6 female mice
(Envigo) at 8 weeks of age received subcutaneous injections in both flanks of $50 \mu \mathrm{g} \mathrm{MOG}_{40-55}$ peptide in PBS, emulsified in an equal volume of complete Freund's adjuvant, containing $4 \mathrm{mg} / \mathrm{ml}$ of Mycobacterium tuberculosis H37RA (Difco, MI, USA), under ketamine/xylazine at 50 and $5 \mathrm{mg} / \mathrm{kg}$ body weight, respectively. In addition, $250 \mathrm{ng}$ of Pertussis toxin (Sigma) were injected intravenously at day 0 and 2. All animals were weighed and examined daily for welfare and clinical signs, according to the following criteria: 0 , asymptomatic; 0.5 , loss of distal half of tail tone; 1 , loss of entire tail tone; 1.5 , hind limb weakness; 2 , hind limb paralysis; 2.5 , hind limb paraplegia; 3, forelimb weakness; 4, quadriparesis; 4.5, severe quadriparesis; 5, quadriplegia; and 6, death. Clinical follow-up analyzes were performed in a blinded manner by two different observers.

\section{Treatment of EAE with liposome-based immunotherapy}

Liposomes were administered at day 5 and 9 post immunization (p.i.), after EAE induction but before the disease onset (occurring at day 13 p.i.), to evaluate the potential of the therapy in the prevention of EAE development. At this stage, mice did not show clinical signs of the disease but autoimmune destruction was ongoing. Liposomes were injected intraperitoneally (i.p.) at a dose of $1.75 \mathrm{mg}$ of lipid in $100 \mu \mathrm{l}$ of PBS. Mice were treated with liposomes filled with MOG peptide (PSMOG-liposomes, $\mathrm{n}=11$ ) and as controls, with empty PS-liposomes (PS-liposomes, $\mathrm{n}=10$ ), MOG peptide (MOG, $\mathrm{n}=8$ ) or PBS (sham, $\mathrm{n}=13)$. Mice were pooled from two independent experiments.

\section{Analysis of regulatory $\mathrm{T}$ cells}

Regulatory $\mathrm{T}$ cells were assessed in the spleen of PSMOG-liposomes and sham-treated mice at day 15 p.i. This checkpoint was chosen based on the time course of the EAE model. The MOG-specific CD4 ${ }^{+}$ T-cell response takes 7 days approximately after EAE induction. Taking into account that liposomes were injected at days 5 and 9 post-immunization, T-cell analysis was performed 6 days later in order to identify the induced tolerogenic mechanisms. Splenocytes were obtained from mice after mechanical disruption and erythrocyte lysis, and analyzed by flow cytometry (FACSCanto II, BD Biosciences). The amount of classical $\mathrm{CD}^{+}{ }^{+}$regulatory $\mathrm{T}$ cells (Tregs) was determined after membrane staining (CD3-V450, CD4-APC-Cy7; BD Biosciences and CD25-PE; eBioscience, CA, USA), fixation/permeabilization (FoxP3 fixation/permeabilization Concentrate and Diluent; eBioscience) and intracellular staining (FoxP3-APC; eBioscience). $\mathrm{CD}^{+}$ 
Table 1. Liposome features.

\begin{tabular}{|c|c|c|c|c|c|}
\hline Liposome type & $\begin{array}{l}\text { Lipid concentration } \\
(\mathrm{mM})\end{array}$ & Diameter (nm) & $\begin{array}{l}\text { Polydispersity } \\
\text { index (PdI) }\end{array}$ & $\zeta$-potential (mV) & $\begin{array}{l}\text { Encapsulation } \\
\text { efficiency (\%) }\end{array}$ \\
\hline PSMOG-liposomes & $30.01 \pm 0.06$ & $861.29 \pm 130.49$ & $0.32 \pm 0.19$ & $-36.19 \pm 5.32$ & $91.53 \pm 4.10$ \\
\hline
\end{tabular}

type $1 \mathrm{~T}$ regulatory $(\mathrm{Tr} 1)$ cells were stained with antibodies to CD3-V450, CD4-APC-Cy7, CD49b-FITC and LAG-3-APC (BD Biosciences) [21].

\section{Statistical analysis}

The statistical analysis was performed using the Prism 6.0 software (GraphPad software Inc., CA, USA). For comparisons of unpaired data, a nonparametric Mann-Whitney test was used; for paired comparisons, a nonparametric Wilcoxon test was used. Fisher's exact test was used to compare qualitative variables. For cor- relation between parameters, Spearman's test was used. A p-value $\leq 0.05$ was considered significant.

\section{Results}

Generation \& physicochemical characterization of PS-liposomes loaded with MOG peptide PS-liposomes were prepared with DOPS/DLPC/ $\mathrm{CH}$ at 1:1:1.33 molar ratio to present PS on their surface. Liposome preparations were characterized in terms of diameter, polydispersity index (PdI), stability $(\zeta$-potential) and efficiency of peptide encapsula-
(A)

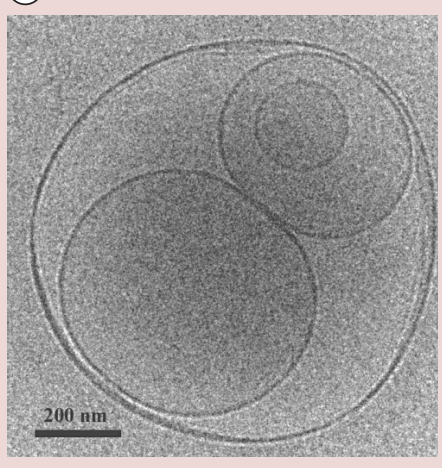

(C)
(B)

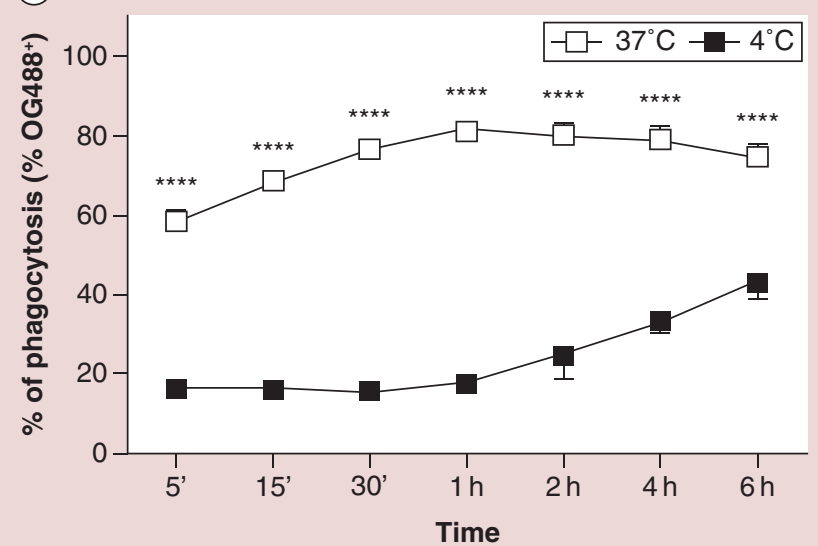

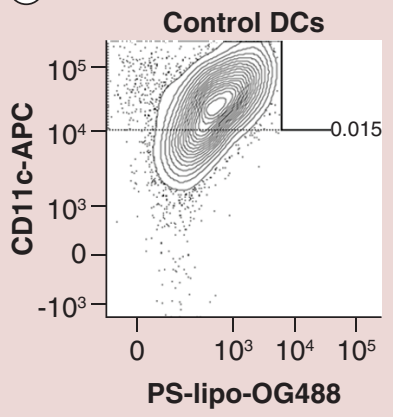
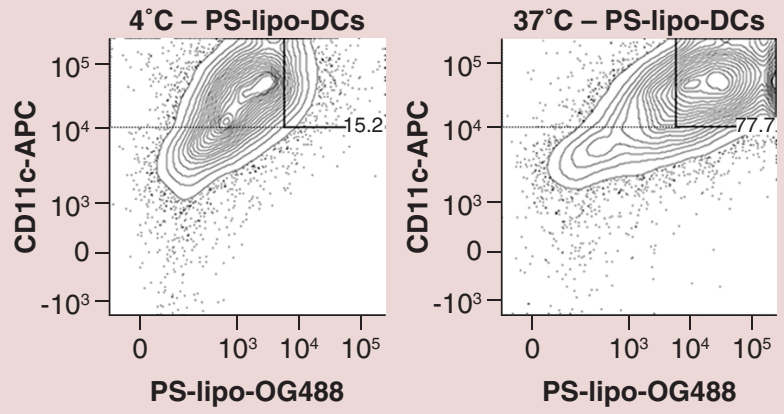

Figure 1. Liposomes are phagocytosed by dendritic cells. (A) Cryogenic transmission electron microscopy images of SMOG-liposomes. Bar $=0.2 \mu \mathrm{m}$. (B) Time course of the capture of PS-lipo-OG488 by DCs at $37^{\circ} \mathrm{C}$ (white squares) and at $4{ }^{\circ} \mathrm{C}$ (black squares). Results are mean \pm SD of three independent experiments $(* * * * \mathrm{p}<0.0001$, two-way ANOVA). (C) Flow cytometry contour plots of the uptake of PS-lipo-OG488 (OG488+) by DCs (CD11 $\left.{ }^{\text {high }}\right)$. From left to right, control DCs, DCs co-cultured with PS-lipo-OG488 at 4 and $37^{\circ} \mathrm{C}$. One representative experiment of three (30 min co-culture) is shown. Percentage of liposome capture (thick line) is referred to CD11 chigh $^{\text {cell subset (thin }}$ line).

DC: Dendritic cell; PS-lipo-OG488: OG488-labeled PS-liposome. 
(A)

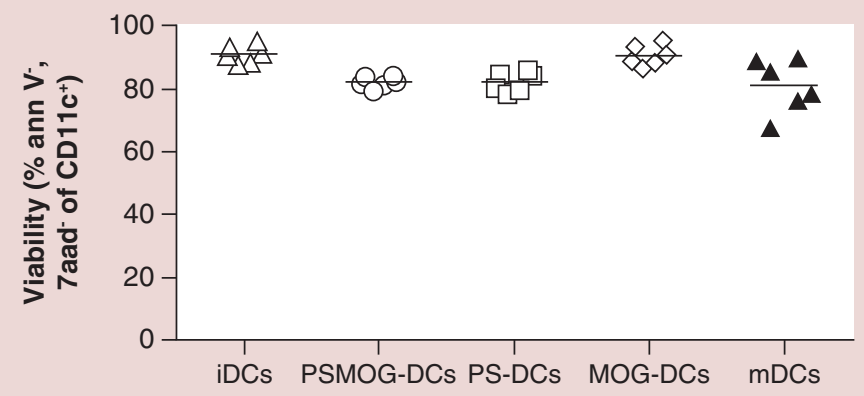

(C)

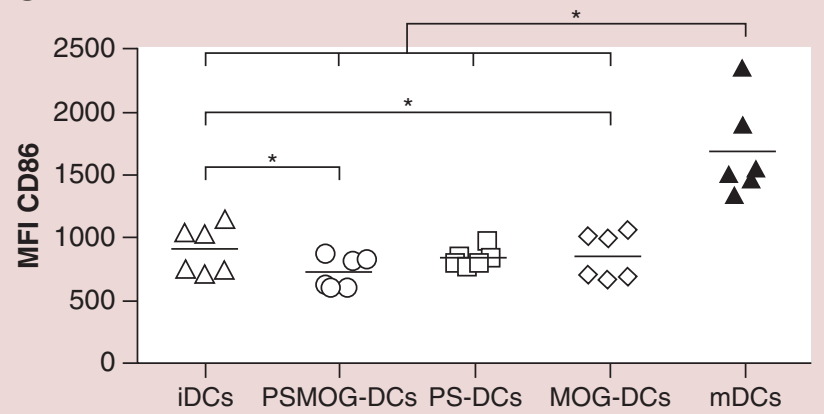

(B)

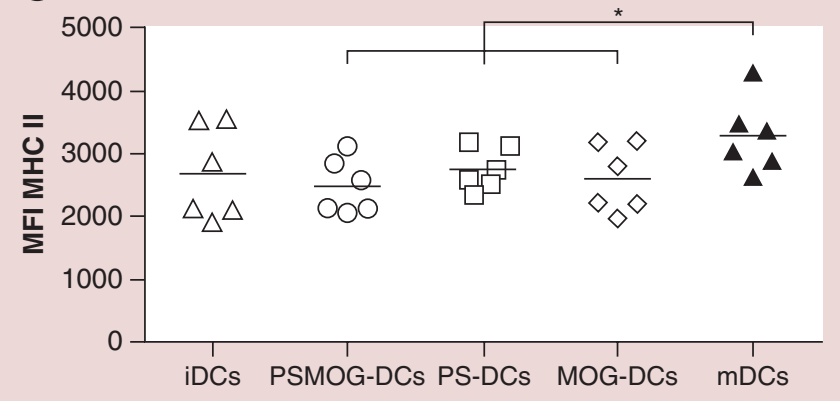

(D)

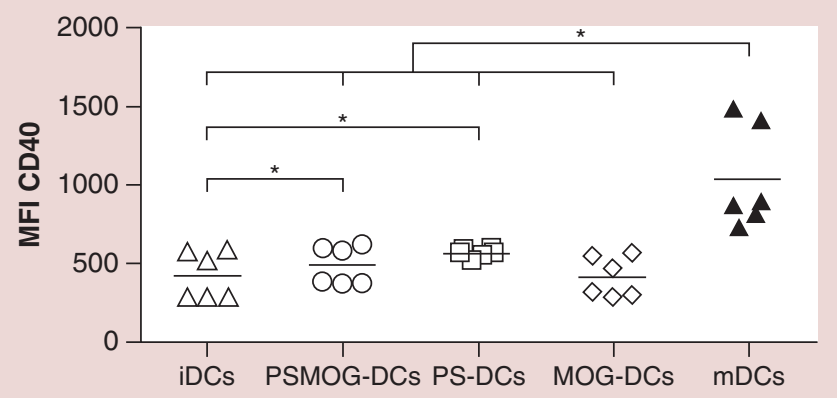

Figure 2. Effect of the capture of phosphatidylserine-liposomes in dendritic cells phenotype. (A) DCs viability assessed by annexin $V$ and 7-AAD staining. White symbols represent iDCs alone (triangles) and after the capture of MOG peptide (MOG-DCs, rhombus), empty PS-liposomes (PS-DCs, squares) or MOG-filled PS-liposomes (PSMOG-DCs, circles), $24 \mathrm{~h}$ after culture. Black symbols represent mDCs, after 24-h culture with LPS. Lines show the mean of six independent experiments. (B-D) MFI for MHC Class II, CD86 and CD40 membrane expression, respectively, in DCs before and after liposome capture (white symbols) and after exposure to LPS (black symbols). Lines show the mean of six independent experiments. Comparisons between groups showed significant differences $\left({ }^{*} p<0.05\right.$, nonparametric Wilcoxon test).

DC: Dendritic cell; iDC: Immature DC; LPS: Lipopolysaccharide; mDC: Mature DC; MFI: Median of fluorescence intensity; MHC: Major histocompatibility complex; PS: Phosphatidylserine; PS-DC: DC loaded with empty PS-liposomes; PSMOG-DC: DC loaded with PSliposomes encapsulating MOG peptide.

tion when necessary (Table 1). The final lipid concentration of PSMOG-liposomes was $30.01 \pm 0.06 \mathrm{mM}$ $($ mean \pm SD) and displayed a mean diameter of 861.29 $\pm 130.49 \mathrm{~nm}$ with a PdI of $0.32 \pm 0.19$. $\zeta$-potential measurements revealed a net surface charge of $-36.19 \pm$ $5.32 \mathrm{mV}$, and the mean of MOG peptide encapsulation efficiency was $91.53 \pm 4.10 \%$. PSMOG-liposomes presented multivesicular vesicle morphology when cryogenic transmission electron microscopy analysis was performed (Figure 1A). Empty PS-liposomes presented a final lipid concentration of $29.70 \pm 0.46 \mathrm{mM}$, with a mean diameter of $985.33 \pm 144.36 \mathrm{~nm}$, a PdI of $0.35 \pm$ 0.05 and $\zeta$ potential of $-35.40 \pm 8.44 \mathrm{mV}$.

\section{PS-liposomes are efficiently phagocytosed by DCs}

Time course analysis (Figure 1B) revealed that the maximum capture of PS-liposomes by DCs $(81.53 \pm$ $2.05 \%$, mean $\pm \mathrm{SD}$ ) was achieved after $1 \mathrm{~h}$ of co-culture at $37^{\circ} \mathrm{C}$. OG488 fluorescence signal at $37^{\circ} \mathrm{C}$ was higher $(\mathrm{p}<0.0001)$ in each checkpoint when compared with its counterpart at $4^{\circ} \mathrm{C}$. The significantly reduced OG488 signal in the experiments performed at $4^{\circ} \mathrm{C}$ as well as the large diameter of the vesicles $(>500 \mathrm{~nm})$ confirms the active process of phagocytosis by which liposomes are captured. Figure $1 \mathrm{C}$ shows a representative contour plot of PS-lipo-OG488 uptake by DCs after $30 \mathrm{~min}$ of co-culture at 4 and $37^{\circ} \mathrm{C}$.

\section{PS-liposomes preserve an immature phenotype in DCs}

To assess the tolerogenic potential of PSMOG-liposomes, we analyzed their effect in DCs phenotype. Viability of DCs after the capture of PSMOG-liposomes was always $>80 \%$ (Figure $2 \mathrm{~A}$ ). Control DCs, cocultured with empty PS-liposomes or MOG peptide, behaved similarly to iDCs in terms of viability.

Afterward, the effect of liposome capture in DCs phenotype in terms of costimulation and antigen presentation was examined. Liposome capture did not affect the expression of MHC II in comparison to iDCs (Figure 2B). The expression of CD86 in iDCs decreased 


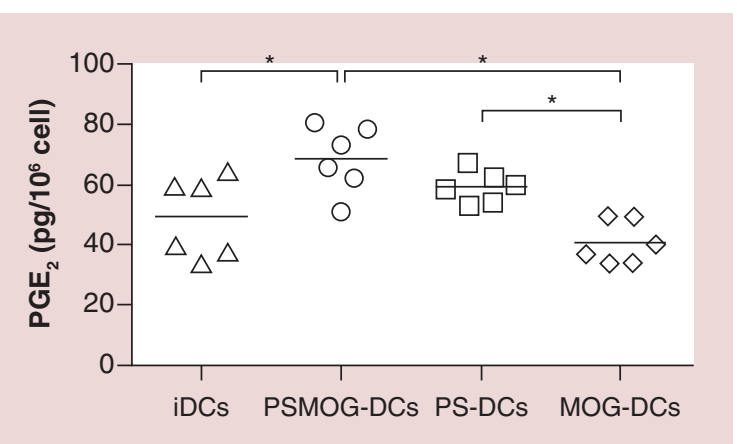

Figure 3. Dendritic cells produce $\mathrm{PGE}_{2}$ after phagocytosis of PS-rich liposomes loaded with myelin-oligodendrocyte glycoprotein. Quantification of $\mathrm{PGE}_{2}$ released by $\mathrm{iDCs}$ in culture medium (triangles), co-cultured with PSMOG-liposomes (circles), empty PSliposomes (squares) or MOG peptide (rhombus) after $24 \mathrm{~h}$ of culture. Data are represented as pg/106 cells. Lines show the mean of six independent experiments. Comparisons between groups showed significant differences ( ${ }^{*} p<0.05$, nonparametric Wilcoxon test). DC: Dendritic cell; iDC: Immature DC; MOG: Myelinoligodendrocyte glycoprotein peptide 40-55; PGE2: Prostaglandin E2; PS: Phosphatidylserine; PSMOG: PS-rich liposome loaded with myelinoligodendrocyte glycoprotein.

after PSMOG-liposomes capture $(\mathrm{p}<0.05)$, and the expression of CD40 in iDCs increased after PS- or PSMOG-liposomes capture $(\mathrm{p}<0.05)$ (Figure $2 \mathrm{C} \& \mathrm{D})$. The expression of CD86, CD40 and MHC class II molecules in DCs after uptake of PSMOG-liposomes was always significantly lower than the observed in mature DCs. These changes suggest that iDCs acquired a semimature phenotype after capture of PSMOG-liposomes, a feature of tolerogenic DCs. Control DCs, co-cultured with empty PS-liposomes or MOG peptide, behaved similarly to iDCs in terms of phenotype.

Based on our previous results, $\mathrm{PGE}_{2}$ production by DCs after PS-liposomes uptake was assessed in culture supernatants. The concentration of $\mathrm{PGE}_{2}$ was significantly increased in PSMOG-DCs in comparison to iDCs and MOG-DCs $(\mathrm{p}<0.05)$, as well as in PS-DCs when compared with MOG-DCs $(\mathrm{p}<0.05)$ (Figure 3).
PS-liposomes filled with MOG peptides reduce EAE severity

Mice were treated with two doses of PSMOG-liposomes at days 5 and 9 p.i. to prevent the development of EAE. As expected, all mice from the sham-control group $(\mathrm{n}=13)$ developed EAE with a maximum score of $2.83 \pm 1.94$ (mean \pm SD) (Table 2). The treatment with PSMOG-liposomes $(\mathrm{n}=11)$ resulted in a significant reduction of maximum disease score, this being of $1.41 \pm 1.59$, in comparison to empty PS-liposomes group $(\mathrm{n}=10)(\mathrm{p}<0.01)$, whose disease score was 3.90 \pm 1.70 , or to MOG peptide group $(n=8)$, whose disease score was $2.38 \pm 1.77$. The incidence of the disease was lower in PSMOG-liposomes treated group (45.45\%) in comparison to empty PS-liposomes treated (100\%, $\mathrm{p}<0.05)$ and sham group $(92.31 \%, \mathrm{p}<0.05)$, and slightly lower than the MOG-treated group (75\%). Mice treated with PSMOG-liposomes showed a lower cumulative EAE score $(14.36 \pm 17.89)$ than mice treated with empty PS-liposomes $(45.60 \pm 23.61)$ or sham $(42.19 \pm 24.99)$ groups $(\mathrm{p}<0.01)$. Moreover, the onset of EAE was at day $16.00 \pm 4.56$ p.i. in PSMOGliposomes treated group, $14.80 \pm 2.70$ in PS-liposomes treated group, $15.50 \pm 2.35$ in MOG peptide treated group and $13.17 \pm 4.73$ in sham group. Finally, mice treated with PSMOG-liposomes showed a daily EAE score significantly lower than sham-control group and PS-liposomes treated group ( $\mathrm{p} \leq 0.05)$ (Figure 4A). In addition, mice treated with PSMOG-liposomes did not suffer a decrease in their body weight as mice from PS-liposomes, MOG peptide or sham groups endured $(\mathrm{p}<0.001)$ (Figure 4B)

\section{PSMOG-liposomes effect on T-cell subtypes}

To test the effect of autoantigen-loaded PS-liposomes administration on regulatory T-cell subsets, intracellular FoxP3 staining was carried out on splenocytes of sham $(n=12)$ and PSMOG-liposomes $(n=6)$ treated animals at day 15 p.i. Looking at $\mathrm{CD}^{+} \mathrm{T}$ cells, the treatment with PSMOG-liposomes significantly decreased the percentage of CD25 ${ }^{+} \mathrm{FoxP}^{+} \mathrm{T}$ cells when compared with sham group, that being of 10.95

Table 2. Clinical features of experimental autoimmune encephalomyelitis in treated and control
mice.
\begin{tabular}{|lllll|}
\hline Treatment & Sham & MOG & PS-liposomes & PSMOG-liposomes \\
Incidence & $12 / 13(92.31 \%)^{*}$ & $6 / 8(75 \%)$ & $10 / 10(100 \%)$ & $5 / 11(45.45 \%)$ \\
\hline Maximum score & $2.83 \pm 1.94$ & $2.38 \pm 1.77$ & $3.90 \pm 1.70 * *$ & $1.41 \pm 1.59$ \\
Cumulative score & $42.19 \pm 24.99 * *$ & $25.88 \pm 21.48$ & $45.60 \pm 23.61 * *$ & $14.36 \pm 17.89$ \\
\hline Onset day & $13.17 \pm 4.73$ & $15.50 \pm 2.35$ & $14.80 \pm 2.70$ & $16.00 \pm 4.56$ \\
\hline $\begin{array}{l}\text { Data are expressed as mean } \pm \text { standard deviation. Comparisons between PSMOG-liposomes treated group versus sham and PS-liposomes } \\
\text { treated groups showed significant differences (*p < 0.05, Fisher's Exact test; **p < 0.01, Mann-Whitney test). } \\
\text { PSMOG-liposome: PS-rich liposome loaded with myelin-oligodendrocyte glycoprotein. }\end{array}$ & \\
\hline
\end{tabular}


(A)

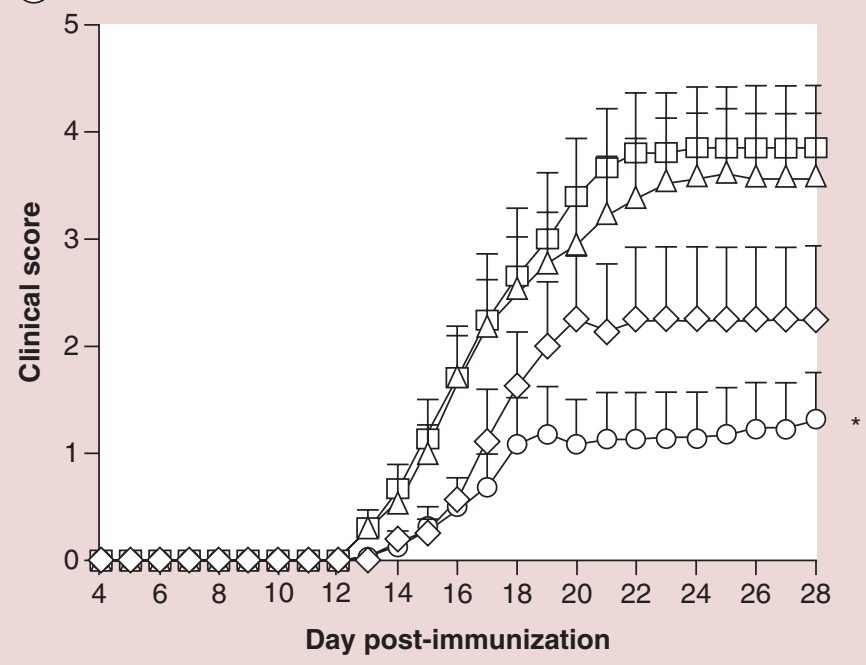

(B)

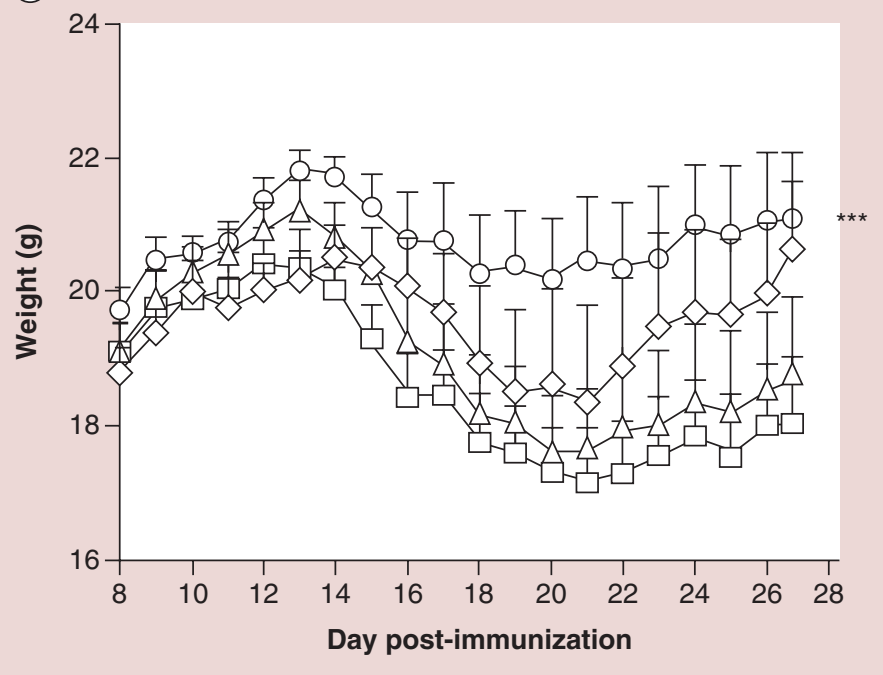

Figure 4. PS-rich liposomes loaded with myelin-oligodendrocyte glycoprotein prevent the development of experimental autoimmune encephalomyelitis. (A) Clinical score of experimental autoimmune encephalomyelitis performed daily for welfare and clinical status as well as neurological signs according to the criteria: 0 , asymptomatic; 0.5 , loss of distal half of tail tone; 1 , loss of entire tail tone; 1.5 , hind limb weakness; 2 , hind limb paralysis; 2.5 , hind limb paraplegia; 3, forelimb weakness; 4, quadriparesis; 4.5, severe quadriparesis; 5, quadriplegia; and 6, death. Follow-up analyzes were performed blindly in mice treated with liposomes containing MOG peptide (PSMOG-liposomes, circles, $n=11$ ), empty liposomes (PS-liposomes, squares, $n=10)$, peptide (MOG, rhombus, $n=8$ ) or PBS (sham, triangles, $n=13$ ). Results are mean \pm standard error of the mean from two independent experiments. Significant differences were found when comparing PSMOG-liposomes group with PS-liposomes and sham groups (* $\leq 50.05$, nonparametric Mann-Whitney test). (B) Mean weight in experimental autoimmune encephalomyelitis-mice after treatment with liposomes containing MOG peptide (PSMOG-liposomes, circles, $n=11$ ), empty liposomes (PS-liposomes, squares, $n=10$ ), peptide (MOG, rhombus, $n=8$ ) or PBS (sham, triangles, $n=13$ ). Results are mean \pm standard error of the mean from two independent experiments. Significant differences were found when comparing PSMOG-liposomes treated group with all control groups $(* * * p<0.001$, nonparametric Mann-Whitney test).

PSMOG-liposome: PS-rich liposome loaded with myelin-oligodendrocyte glycoprotein.

$\pm 3.48 \%($ mean $\pm \mathrm{SD})$ and $14.69 \pm 2.67 \%$, respectively $(\mathrm{p}<0.05)$. However, we noted that the percentage of $\mathrm{CD}^{2} 5^{+}$FoxP3- $\mathrm{T}$ cells in PSMOG-liposomes group increased when compared with their sham counterparts, that being of $14.83 \pm 5.47 \%$ and $9.65 \pm 2.84 \%$, respectively $(\mathrm{p}<0.05)$ (Figure 5A). Indeed, the percentage of $\mathrm{CD}_{25} 5^{+}$FoxP3- $\mathrm{T}$ cells in PSMOG-liposomes treated mice correlated inversely with the clinical score at day 15 p.i. (Spearman's $r=-0.9258, p<0.0001$ ), as shown in Figure 5B, but no correlation was observed between these parameters in sham group (data not shown). Nevertheless, the number of $\mathrm{CD}^{2} 5^{+}$Foxp $3^{+}$ cells tended to decrease in PSMOG-liposomes group $\left(6.31 \times 10^{5} \pm 2.03 \times 10^{5}\right)$, when compared with sham group $\left(11.05 \times 10^{5} \pm 0.76 \times 10^{5}\right)$, although differences are not statistically significant. Regarding the absolute number of $\mathrm{CD}_{25} 5^{+}$FoxP3- $\mathrm{T}$ cells, similar results were obtained in PSMOG-liposomes treated group $(9.13 \times$ $\left.10^{5} \pm 4.58 \times 10^{5}\right)$ and sham group $\left(8.95 \times 10^{5} \pm 1.71\right.$ $\left.\times 10^{5}\right)$. With these results, the expression of $\mathrm{CD}^{+} \operatorname{Tr} 1$ cell markers CD 49b and LAG-3 was next examined on the splenocytes of the sham and PSMOG-liposomes groups by analyzing their median of fluorescence intensity. Although there were no statistically significant differences in the expression of CD49b between sham $(90.18 \pm 11.49$, mean \pm SD) and treated $(95.12 \pm$ 13.84) groups, a tendency for this marker to increase in PSMOG-liposomes treated mice was observed (Figure 5C). Supporting this tendency, LAG-3 expression was significantly increased in PSMOG-liposomes treated mice $(387.5 \pm 88.53)$ when compared with sham group $(304.6 \pm 54.41)(\mathrm{p} \leq 0.05)$.

\section{Discussion}

It is well known that apoptotic cell clearance by phagocytes maintains immunological homeostasis and induces antigen-specific immune tolerance [22,23]. In fact, apoptotic mimicry is a strategy to induce tolerance, lost in autoimmune diseases. In this sense, several approaches have been developed [7,20,24]. We have recently shown that apoptotic mimicry by means of liposomes - rich in PS and encapsulating autoantigens - arrest autoimmunity in T1D [8]. Therefore, we hypothesized that by replacing the encapsulated autoantigen, PS-liposomes would show therapeutic effect for other autoimmune diseases. 
We selected MS to assess therapeutic versatility of liposomes. In the present study, we have validated the efficacy of PS-liposomes in the experimental model of the disease. MS is one of the most frequent neurological disorders [25], and epidemiological studies have highlighted an increasing rate of prevalence of the disease. Prevention and cure of MS is not yet possible. Some therapies can slow the progress of the disease and manage the symptoms, but they cause important side effects.

The growing field of micro and nanotechnology has enabled new strategies for MS and other autoim- mune diseases to be tested in experimental models. Nanoparticles and microparticles have been used to induce T-cell tolerance and as vehicles for anti-inflammatory drugs [26-29], and a few of them have reached the clinic [30]. The relevance of our results relies in the potential of PS-liposomes to develop a platform for the treatment of several autoimmune disorders, using a biomimicry strategy, based on a physiological process such as efferocytosis. In our knowledge, this is the first work in the context of EAE that confirms PS-liposomes as a powerful tool to encapsulate autoantigens, co-delivering a double-signal of tolerance and

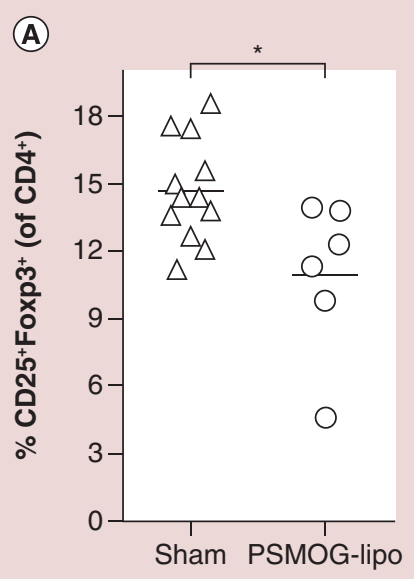

(C)

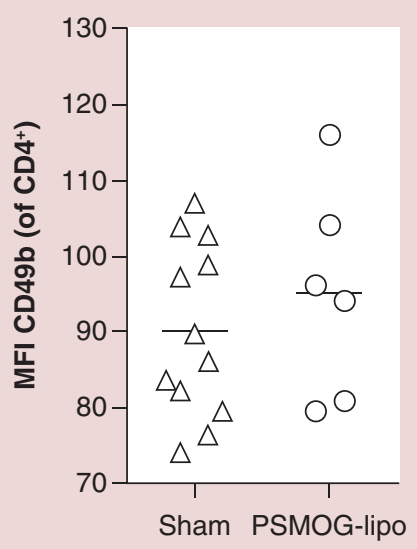

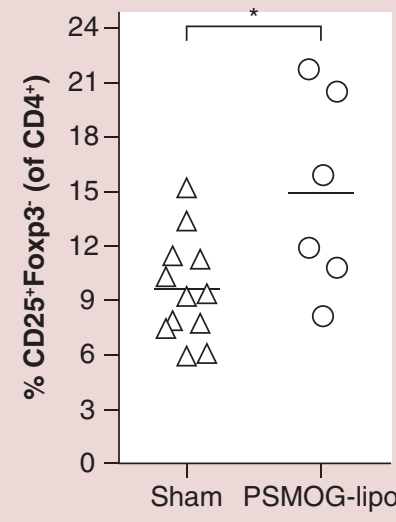
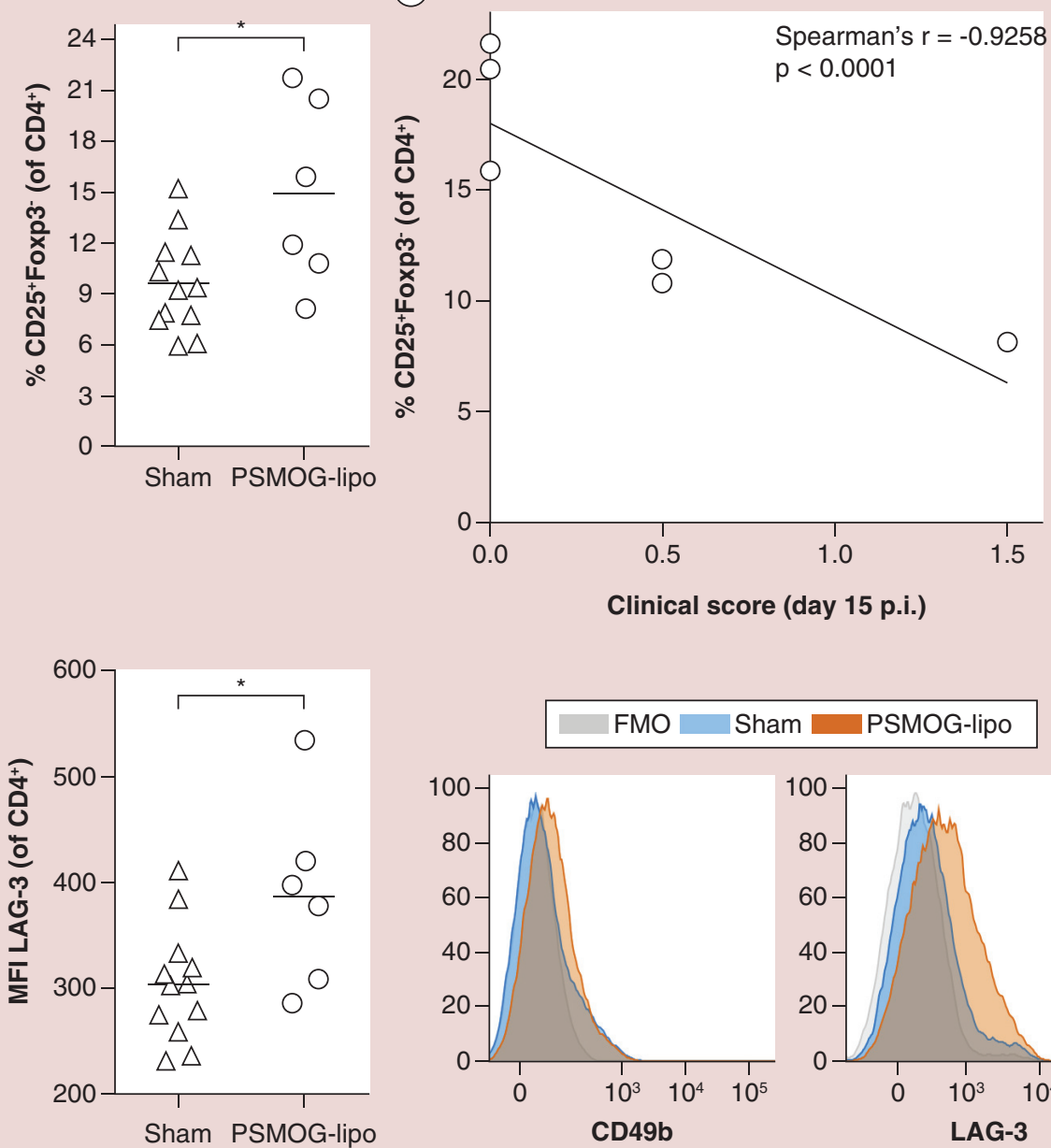

(B)

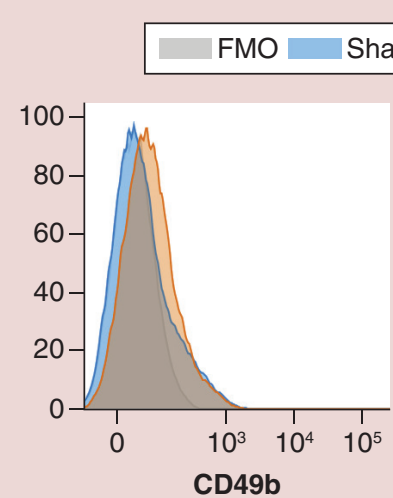

PSMOG-lipo

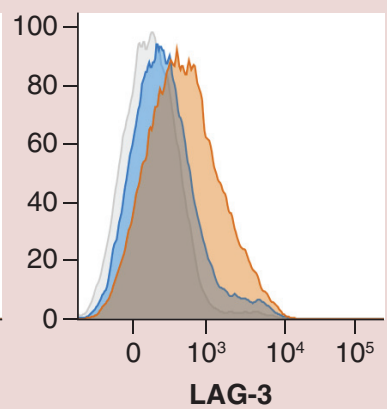

Figure 5. T cell subsets in mice treated with PS-rich liposomes loaded with myelin-oligodendrocyte glycoprotein. (A) Percentage of classical regulatory $\mathrm{T}$ cells (CD25+ $5^{+}$oxP3 ${ }^{+}$cells gated in $\mathrm{CD} 4^{+} \mathrm{T}$ cells) and $\mathrm{CD} 25^{+}$FoxP3- cells (gated in CD4+ ${ }^{+}$cells) in the spleen of PSMOG-liposomes treated mice (circles, $n=6$ ) or sham control group (triangles, $n=12$ ) at day 15 p.i. Lines show the mean of 6-12 mice. Comparisons between groups showed significant differences ( $p<0.05$, nonparametric Mann-Whitney test). (B) Correlation between clinical score at day 15 p.i. and percentage of CD25 $5^{+}$FoxP3- cells (gated in CD4 $4^{+} \mathrm{T}$ cells) in PSMOG-liposomes treated mice (**** $<0.0001$, Spearman's correlation analysis). (C) Left panel: MFI of CD49b and LAG-3 membrane expression in CD4+ T cells from PSMOG-liposomes treated mice or sham control group at day 15 p.i. Lines show the mean of 6-12 mice. Comparisons between groups showed significant differences ( $p<0.05$, nonparametric Mann-Whitney test). Right panel: Histogram of one representative experiment is shown for CD49b and LAG3 expression in mice treated with PSMOG-liposomes (orange) or sham (blue).

FMO: Fluorescence minus one; MFI: Median of fluorescence intensity; P.i.: Post immunization. 
specificity to arrest autoimmune reactions in a synergistic, effective and safe manner. Liposomes provide an effective tolerogenic-signal induction with additional advantages. They are biocompatible, completely biodegradable, nontoxic, nonimmunogenic, suitable for encapsulation of peptides with varying solubility and low cost [31]. Moreover, they protect the encapsulated drug from the external environment and exhibit flexibility to be coupled with site-specific ligands (e.g., PS and its receptor in DCs).

Various types of liposomes are being used as vehicles [32] for anticancer drugs, vaccines and anti-inflammatory agents $[2,33,34]$. In this sense, liposomes have been used for the treatment of MS but PS was not included in their composition [35]. PS is the main 'eat me' and 'tolerate me' signal of the apoptotic cell membrane, which allows recognition and phagocytosis by antigen presenting cells such as DCs [36]. Apoptotic cell signaling prevents maturation of DCs and promotes autoantigen presentation in a tolerogenic manner, to induce specific tolerance rather than autoimmunity [7]. Conversely, a high rate of apoptosis and/or defects in their clearance contribute to autoimmunity, since apoptotic cells turn into secondary necrotic cells, releasing proinflammatory signals that enable maturation of DCs and immunogenic presentation of autoantigens. In this sense, neuronal apoptosis is induced in cultures exposed to cerebrospinal fluid from MS patients [37], pointing to a relevant role of apoptosis in the disease progression. These data fit well with the herein reported effect of PSMOG-liposomes in the re-establishment of self-tolerance in the EAE model.

The morphological and physicochemical features of the liposomes are key to provide them with this tolerogenic potential. To validate the liposome effect reported in experimental T1D in MS, we designed EAE-adapted liposomes to be similar to those used for T1D. In this work, we demonstrate the capacity of this already-published lipid composition to prevent a completely different autoimmune disease by replacing the relevant autoantigen in T1D with the corresponding peptide for EAE and keeping lipid concentration, diameter and charge. This conceptual innovation allows us to consider this strategy as a potential platform for the reestablishment of immunological tolerance. In vitro experiments demonstrate that liposomes are phagocytosed by DCs from another experimental model (EAE) inducing the same phenotypic and functional changes in DCs than in the T1D model [8].

In vitro experiments revealed crucial immunological consequences of the capture of PSMOG-liposomes by iDCs. First, liposomes were rapidly, actively and safely captured through phagocytosis by DCs, as demonstrated by co-culture experiments at 37 and $4^{\circ} \mathrm{C}[38,39]$.
Endocytic processes involve the internalization of large-sized particles or a large volume of the extracellular bulk. These are termed phagocytosis and macropinocytosis, respectively: phagocytosis refers to the transport of large particles (>250 $\mathrm{nm}$ in diameter) by immune system specialized cells (macrophages, DCs and neutrophils), while pinocytosis refers to ingestion of fluids and solutes (about $100 \mathrm{~nm}$ in diameter) by mammalian cells [40]. In this sense, the large size of the here reported liposomes - diameter greater than $500 \mathrm{~nm}-$ and the presence of PS as an 'eat-me' signal promote active phagocytosis by DCs $[8,41]$. Second, after liposome engulfment, DCs increased CD40 membrane expression and decreased CD86, and maintained low membrane expression of MHC class II expression, features of tolerogenic DCs. Interestingly, PSMOGliposomes uptake induced the secretion of $\mathrm{PGE}_{2}$, an essential mediator in the maintenance of tolerance after efferocytosis [42]. These results are in agreement with our previous study, using specific PS-liposomes for T1D.

Importantly, liposome treatment was successful at arresting autoimmunity in vivo and greatly ameliorating clinical symptoms in treated mice in terms of severity, incidence and disease onset. As expected, no effect was observed when mice received empty PSliposomes. As described from previous studies [43], treatment with MOG peptide resulted in an amelioration of the disease, and it was in an antigen-specific manner [44]. However, the effect of MOG peptide appeared to be minor than the induced by PSMOGliposomes, which reflect the joint action of both PS and MOG. Also, combined therapy with MOG peptide and other compounds such as vitamin D or Rapamycin displays synergisms in ameliorating $\operatorname{EAE}[45,46]$. Here we show the antigen specificity of the treatment, as empty PS-liposomes do not provide protection in front EAE symptoms whereas treatment with liposomes filled with MOG peptide clearly resulted in a strong protection from disease. This immunotherapy is safe and even more biologically effective than the treatment with MOG peptide because PS-liposomes mimic apoptotic cells, thus providing specificity and tolerogenic signals to DCs. These results are consistent with previous data obtained in T1D studies, showing a requirement for $\beta$-cell autoantigens in liposomes to arrest autoimmunity to islet cells [8].

The mechanism of action of the herein reported immunotherapy should be grounded on the promotion of regulatory mechanisms by DCs after liposome capture. The decrease in frequency of $\mathrm{CD} 25^{+}$FoxP3 ${ }^{+}$ $\mathrm{CD}^{+} \mathrm{T}$ cells as well as in absolute numbers (although nonstatistically significant) is consistent with an increase in frequency of CD25+ FoxP3- $\mathrm{CD} 4^{+} \mathrm{T}$ cells. 
However, absolute numbers of $\mathrm{CD} 25^{+}$FoxP3- $\mathrm{CD}^{+}$ $\mathrm{T}$ cells are not altered by liposomes, suggesting that additional regulatory mechanisms may be involved. These results are in line with those obtained in experimental autoimmune T1D [8]. In fact, CD25 ${ }^{+}$FoxP3 $\mathrm{CD}^{+}{ }^{+} \mathrm{T}$-cell frequency is higher in treated mice with minor EAE clinical score, so it is reasonable to speculate that these cells have a regulatory function and can belong to a subset of antigen-specific regulatory $\mathrm{T}$ lymphocytes. For instance, we cannot rule out an increase in antigen-specific Tr1 cells after PSMOGliposomes treatment, based on the CD49b and LAG-3 expression profile [21], as reported in other nanotherapies aimed to restore self-tolerance [8,29]. LAG-3 marker is significantly increased in mice treated with PSMOG-liposomes and, although statistically nonsignificant, CD49b shows a tendency to increase in treated mice. In this sense, LAG-3 expression is sufficient to confer regulatory activity in $\mathrm{CD}^{+}{ }^{+} \mathrm{T}$ cells $[47]$, upholding the possible generation of $\operatorname{Tr} 1$ with the described immunotherapy.

Altogether our results highlight the potential of a PS-liposomes platform for the prevention and reversion of autoimmune diseases, with advantages in terms of production feasibility, stability, safety, biocompatibility, costs and customization for different diseases, as long as the autoantigen is identified. The here reported work validates PS-liposomes as a powerful tool to encapsulate autoantigens, co-delivering a double-signal of tolerance and specificity to arrest autoimmunity in a synergistic and effective manner.

\section{Conclusion}

PS-liposomes encapsulating self-peptides are sphereshaped microvesicles designed to achieve tolerogenic delivery of autoantigens into antigen presenting cells in order to arrest autoimmunity. PS-liposomes loaded with a MS autoantigen (MOG) were prepared and tested in an experimental model of the disease, the EAE-mouse model. PSMOG-liposomes were efficiently captured by DCs, inducing a tolerogenic phenotype, and ceasing the autoimmune reaction in EAE-mice, in the same way that PS-liposomes customized for T1D do. This work validates PSliposomes as a powerful tool for the re-establishment of tolerance and for the treatment of different autoimmune diseases.

\section{Supplementary data}

To view the supplementary data that accompany this paper please visit the journal website at: www.futuremedicine.com/ doi/full/10.2217/nnm-2016-0410

Financial \& competing interests disclosure

This work was supported by a grant from the Spanish Government (FIS PI15/00198) co-financed with the European Regional Development funds (FEDER) and by CERCA Programme, Generalitat de Catalunya. CIBER of Diabetes and Associated Metabolic Diseases (CIBERDEM) is an initiative from Instituto de Salud Carlos III. This work has been supported by positive discussion through A FACTT network (Cost Action BM1305: www.afactt.eu). COST is supported by the EU Framework Programme Horizon 2020. S Rodriguez-Fernandez and J Na-

\section{Summary points}

\section{Background}

- The prevalence of autoimmunity is on the rise, and there is no cure for any autoimmune disease, caused by the loss of tolerance to self.

- One of the mechanisms to maintain self-tolerance is the efficient removal of apoptotic cells. For this reason, a liposome-based antigen-specific immunotherapy was generated.

- Liposomes that mimic apoptotic $\beta$ cells prevent Type 1 diabetes through specific and definitive re-establishment of tolerance.

Aim

- Having proved that liposomes rich in phosphatidylserine (PS) and loaded with autoantigens were effective for Type 1 diabetes, our aim was to validate the immunotherapy in other autoimmune disease: multiple sclerosis. Results

- PS-Liposomes loaded with a multiple sclerosis autoantigen are efficiently phagocytosed by dendritic cells and induce tolerogenic features in dendritic cells.

- After post immunization administration, MOG-loaded PS-liposomes reduce the incidence and severity of experimental autoimmune encephalomyelitis, and delay the onset.

- The arrest of the autoimmune attack correlates with an increase in the CD25+ FoxP3- CD4+ T-cell subset. Conclusion

- This is the first work in the context of experimental autoimmune encephalomyelitis that confirms PSliposomes as a powerful tool to encapsulate autoantigens, co-delivering a double-signal of tolerance and specificity to arrest autoimmunity in a synergistic, effective and safe manner. Autoantigen-loaded PSliposomes are candidates for immunotherapy to induce self-tolerance, with high potential to operate as a platform for autoimmune diseases. 
varro-Barriuso are supported by grants from the AGAUR, Generalitat de Catalunya. Special thanks to MA Cardus and her family for their generous donation. The funders had no role in study design, data collection and analysis, decision to publish or preparation of the manuscript. The authors have no other relevant affiliations or financial involvement with any organization or entity with a financial interest in or financial conflict with the subject matter or materials discussed in the manuscript apart from those disclosed.

No writing assistance was utilized in the production of this manuscript.

\section{References}

Papers of special note have been highlighted as: $\bullet$ of interest; •• of considerable interest

1 Akbarzadeh A, Rezaei-Sadabady R, Davaran S et al. Liposome: classification, preparation, and applications. Nanoscale Res. Lett. 8(1), 102 (2013).

2 Chang HI, Yeh MK. Clinical development of liposomebased drugs: formulation, characterization, and therapeutic efficacy. Int. J. Nanomedicine 7, 49-60 (2012).

- Summary of the preclinical and clinical data related to liposomal formulations and their physicochemical properties.

3 Jiao D, Geng J, Loh XJ, Das D, Lee TC, Scherman OA. Supramolecular peptide amphiphile vesicles through hostguest complexation. Angew. Chem. Int. Ed. Engl. 51(38), 9633-9637 (2012).

4 Hwang JY, Li Z, Loh XJ. Small molecule therapeuticloaded liposomes as therapeutic carriers: from development to clinical applications. RSC Adv. 6(74), 70592-70615 (2016).

5 Fenske DB, Cullis PR. Liposomal nanomedicines. Expert Opin. Drug Deliv. 5(1), 25-44 (2008).

6 Watson DS, Endsley AN, Huang L. Design considerations for liposomal vaccines: influence of formulation parameters on antibody and cell-mediated immune responses to liposome associated antigens. Vaccine 30(13), 2256-2272 (2012).

7 Steinman RM, Turley S, Mellman I, Inaba K. The induction of tolerance by dendritic cells that have captured apoptotic cells. J. Exp. Med. 191(3), 411-416 (2000).

-• Describes the role of dendritic cells in inducing immunological tolerance after the engulfment of apoptotic bodies. Phagocytosis of apoptotic bodies prevents dendritic cell maturation to ensure tolerance to self.

8 Pujol-Autonell I, Serracant-Prat A, Cano-Sarabia M et al. Use of autoantigen-loaded phosphatidylserine-liposomes to arrest autoimmunity in type 1 diabetes. PLoS ONE 10(6), e0127057 (2015).

-. Describes the use of liposomes rich on phosphatidylserine encapsulating insulin peptides to arrest autoimmunity in experimental Type 1 diabetes. This is the first report on Type 1 diabetes prevention using liposomes.

\section{Ethical conduct of research}

The authors state that they have obtained appropriate institutional review board approval and have followed the principles outlined in the Declaration of Helsinki for animal experimental investigation.

\section{Open access}

This work is licensed under the Creative Commons Attribution-NonCommercial 3.0 Unported License. To view a copy of this license, visit http://creativecommons.org/licenses/bync-nd/3.0/

9 Chen X, Doffek K, Sugg SL, Shilyansky J. Phosphatidylserine regulates the maturation of human dendritic cells. J. Immunol. 173(5), 2985-2994 (2004).

10 Wang L, Wang FS, Gershwin ME. Human autoimmune diseases: a comprehensive update. J. Intern. Med. 278(4), 369-395 (2015).

11 Okada H, Kuhn C, Feillet H, Bach JF. The 'hygiene hypothesis' for autoimmune and allergic diseases: an update. Clin. Exp. Immunol. 160(1), 1-9 (2010).

12 Mcfarland HF, Martin R. Multiple sclerosis: a complicated picture of autoimmunity. Nat. Immunol. 8(9), 913-919 (2007).

13 Sospedra M, Martin R. Immunology of multiple sclerosis. Semin. Neurol. 36(2), 115-127 (2016).

14 Steinman L. Immunology of relapse and remission in multiple sclerosis. Annu. Rev. Immunol. 32, 257-281 (2014).

15 Cross AH, Naismith RT. Established and novel diseasemodifying treatments in multiple sclerosis. J. Intern. Med. 275(4), 350-363 (2014).

16 Rangachari M, Kuchroo VK. Using EAE to better understand principles of immune function and autoimmune pathology. J. Autoimmun. 45, 31-39 (2013).

17 Mansilla MJ, Selles-Moreno C, Fabregas-Puig S et al. Beneficial effect of tolerogenic dendritic cells pulsed with MOG autoantigen in experimental autoimmune encephalomyelitis. CNS Neurosci. Ther. 21(3), 222-230 (2015).

- Description of the experimental model for the study of multiple sclerosis, the experimental autoimmune encephalomyelitis mouse, and experiments performed with dendritic cell therapy to decrease clinical severity of disease.

18 Pugliese A. Central and peripheral autoantigen presentation in immune tolerance. Immunology 111(2), 138-146 (2004).

19 Harel-Adar T, Ben Mordechai T, Amsalem Y, Feinberg MS, Leor J, Cohen S. Modulation of cardiac macrophages by phosphatidylserine-presenting liposomes improves infarct repair. Proc. Natl Acad. Sci. USA 108(5), 1827-1832 (2011).

20 Marin-Gallen S, Clemente-Casares X, Planas R et al. Dendritic cells pulsed with antigen-specific apoptotic bodies prevent experimental type 1 diabetes. Clin. Exp. Immunol. 160(2), 207-214 (2010). 
- First approach of our group developed to arrest autoimmunity using apoptotic cells. These experiments were the basis to design liposomes for apoptotic mimicry.

21 Gagliani N, Magnani CF, Huber S et al. Coexpression of CD 49b and LAG-3 identifies human and mouse T regulatory type 1 cells. Nat. Med. 19(6), 739-746 (2013).

22 Nagata S, Hanayama R, Kawane K. Autoimmunity and the clearance of dead cells. Cell 140(5), 619-630 (2010).

23 Poon IK, Lucas CD, Rossi AG, Ravichandran KS. Apoptotic cell clearance: basic biology and therapeutic potential. Nat. Rev. Immunol. 14(3), 166-180 (2014).

24 Miller SD, Turley DM, Podojil JR. Antigen-specific tolerance strategies for the prevention and treatment of autoimmune disease. Nat. Rev. Immunol. 7(9), 665-677 (2007).

25 Milo R, Kahana E. Multiple sclerosis: geoepidemiology, genetics and the environment. Autoimmun. Rev. 9(5), A387-A394 (2010).

26 Hunter Z, Mccarthy DP, Yap WT et al. A biodegradable nanoparticle platform for the induction of antigen-specific immune tolerance for treatment of autoimmune disease. ACS Nano 8(3), 2148-2160 (2014).

27 Turjeman K, Bavli Y, Kizelsztein P et al. Nano-drugs based on nano sterically stabilized liposomes for the treatment of inflammatory neurodegenerative diseases. PLoS ONE 10(7), e0130442 (2015).

28 Getts DR, Martin AJ, Mccarthy DP et al. Microparticles bearing encephalitogenic peptides induce T-cell tolerance and ameliorate experimental autoimmune encephalomyelitis. Nat. Biotechnol. 30(12), 1217-1224 (2012).

29 Clemente-Casares X, Blanco J, Ambalavanan P et al. Expanding antigen-specific regulatory networks to treat autoimmunity. Nature 530(7591), 434-440 (2016).

30 Lutterotti A, Yousef S, Sputtek A et al. Antigen-specific tolerance by autologous myelin peptide-coupled cells: a Phase I trial in multiple sclerosis. Sci. Transl. Med. 5(188), 188ra175 (2013).

31 Kapoor B, Singh SK, Gulati M, Gupta R, Vaidya Y. Application of liposomes in treatment of rheumatoid arthritis: quo vadis. ScientificWorldJournal 2014, 978351 (2014).

32 Gharagozloo M, Majewski S, Foldvari M. Therapeutic applications of nanomedicine in autoimmune diseases: from immunosuppression to tolerance induction. Nanomedicine 11(4), 1003-1018 (2015).

33 Van Der Valk FM, Van Wijk DF, Lobatto ME et al. Prednisolone-containing liposomes accumulate in human atherosclerotic macrophages upon intravenous administration. Nanomedicine 11(5), 1039-1046 (2015).

34 Van Den Hoven JM, Van Tomme SR, Metselaar JM, Nuijen B, Beijnen JH, Storm G. Liposomal drug formulations in the treatment of rheumatoid arthritis. Mol. Pharm. 8(4), 1002-1015 (2011).

35 Lomakin Y, Belogurov A Jr, Glagoleva I et al. Administration of myelin basic protein peptides encapsulated in mannosylated liposomes normalizes level of serum TNFalpha and IL-2 and chemoattractants CCL2 and CCL4 in multiple sclerosis patients. Mediators Inflamm. 2016, 2847232 (2016).

36 Fadok VA, Bratton DL, Henson PM. Phagocyte receptors for apoptotic cells: recognition, uptake, and consequences. J. Clin. Invest. 108(7), 957-962 (2001).

- Report of the role of phosphatidylserine in the recognition and clearance of apoptotic cells by phagocytes.

Phosphatidylserines are key molecules in liposome design and act synergistically with autoantigens in the induction of tolerance, thus arresting autoimmunity.

37 Cid C, Alvarez-Cermeno JC, Regidor I, Plaza J, Salinas M, Alcazar A. Caspase inhibitors protect against neuronal apoptosis induced by cerebrospinal fluid from multiple sclerosis patients. J. Neuroimmunol. 136(1-2), 119-124 (2003).

38 Casley-Smith JR. The dimensions and numbers of small vesicles in cells, endothelial and mesothelial and the significance of these for endothelial permeability. J. Microsc. 90 (3), 251-268 (1969).

39 Marques-Da-Silva C, Burnstock G, Ojcius DM, CoutinhoSilva R. Purinergic receptor agonists modulate phagocytosis and clearance of apoptotic cells in macrophages. Immunobiology 216(1-2), 1-11 (2011).

40 Kumari S, Mg S, Mayor S. Endocytosis unplugged: multiple ways to enter the cell. Cell Res. 20(3), 256-275 (2010).

41 Segawa K, Nagata S. An apoptotic 'Eat Me' signal: phosphatidylserine exposure. Trends Cell Biol. 25(11), 639-650 (2015).

42 Pujol-Autonell I, Ampudia RM, Planas R et al. Efferocytosis promotes suppressive effects on dendritic cells through prostaglandin E2 production in the context of autoimmunity. PLoS ONE 8(5), e63296 (2013).

43 Devaux B, Enderlin F, Wallner B, Smilek DE. Induction of EAE in mice with recombinant human MOG, and treatment of EAE with a MOG peptide. J. Neuroimmunol. 75(1-2), 169-173 (1997).

44 Menges M, Rossner S, Voigtlander C et al. Repetitive injections of dendritic cells matured with tumor necrosis factor alpha induce antigen-specific protection of mice from autoimmunity. J. Exp. Med. 195(1), 15-21 (2002).

45 Chiuso-Minicucci F, Ishikawa LL, Mimura LA et al. Treatment with vitamin D/MOG association suppresses experimental autoimmune encephalomyelitis. PLoS ONE 10(5), e0125836 (2015).

46 Tostanoski LH, Chiu YC, Gammon JM et al. Reprogramming the local lymph node microenvironment promotes tolerance that is systemic and antigen specific. Cell Rep. 16(11), 2940-2952 (2016).

47 Huang CT, Workman CJ, Flies D et al. Role of LAG-3 in regulatory T cells. Immunity 21(4), 503-513 (2004). 